\title{
Polarization gaps and negative group velocity in chiral phononic crystals: Layer multiple scattering method
}

\author{
Huanyang Chen, ${ }^{1,2}$ Kin Hung Fung, ${ }^{1}$ Hongru Ma, ${ }^{2}$ and C. T. Chan ${ }^{1}$ \\ ${ }^{1}$ Department of Physics, Hong Kong University of Science and Technology, Clear Water Bay, Kowloon, Hong Kong, China \\ ${ }^{2}$ Institute of Theoretical Physics, Shanghai Jiao Tong University, Shanghai 200240, People's Republic of China \\ (Received 18 January 2008; revised manuscript received 21 May 2008; published 20 June 2008)
}

\begin{abstract}
We designed chiral phononic crystals and studied their properties by using the layer multiple scattering method. The transmittance curves and the corresponding band structures show that this kind of structure possesses significant polarization gaps. The chiral structures break the symmetry so that the degenerate transverse modes split into a pair of right-hand polarized mode and left-hand polarized mode. The polarization splitting in the low-frequency range is enhanced in systems of large filling ratios. We also demonstrate that chiral structures containing strongly resonant units can induce negative group velocity in elastic waves, and stronger resonance brings a wider band of negative group velocity.
\end{abstract}

DOI: 10.1103/PhysRevB.77.224304

PACS number(s): 63.20.-e, 43.35.+d

\section{INTRODUCTION}

Studies on the properties of photonic and acoustic materials with chiral structures attracted growing interest for their special characteristics, such as negative refraction ${ }^{1,2}$ and polarization gaps. ${ }^{3-7}$ Since the symmetry between the righthand and left-hand circularly polarized waves is broken by chiral structures, the two polarizations travel with different speeds and one of the circular polarizations can potentially be blocked by the polarization gaps (or chiral Bragg gaps). Significant polarization gaps for circularly polarized electromagnetic (EM) waves in spiral photonic crystals have been observed. ${ }^{3}$ Such chiral structures can give exotic thermal radiation properties. ${ }^{8}$ Similar results about polarization gaps of the circularly polarized transverse (CPT) elastic waves were also observed in one kind of materials called piezoelectric continuously twisted structurally chiral medium (PCTSCM) proposed by Lakhtakia. $^{9}$ Recently, a kind of acoustic metamaterials ${ }^{10}$ has been demonstrated to realize the negative refraction in acoustic waves, which is similar to the original idea of negative refraction in EM waves proposed by Veselago. ${ }^{11}$ This kind of metamaterials is named the "doublenegative acoustic metamaterials" due to their simultaneously negative effective bulk modulus and mass density. Such kind of metamaterials possesses a passband of negative group velocity that can lead to negative refraction. In addition to double negativity, there are various mechanisms that can cause negative group velocity and/or negative refraction, such as Bragg scattering and chiral structures. ${ }^{1,7,12}$ The negative refraction induced by Bragg scattering has already realized in acoustic waves. ${ }^{13}$ However, neither negative group velocity nor negative refraction induced by chiral structures has been explicitly demonstrated. There are very few studies, especially experimental ones, which focus on CPT elastic waves. Given that a technique to generate CPT elastic waves was proposed as early as 1964 by Einspruch, ${ }^{14}$ the study of the CPT elastic waves should be given more consideration both experimentally and theoretically, in view of the surge in the interest of artificial wave-functional structures such as phononic crystals. In this paper, we show the existence of polarization gaps (circular Bragg gaps) for CPT elastic waves in a layer-by-layer chiral phononic crystal and demonstrate that there exists a passband of negative group velocity (with controllable bandwidth) when some locally resonant units ${ }^{15}$ are included in the structure. We then have a more complete picture that links special structures to wave properties. We note that an artificial helical structure with one sphere per two-dimensional unit cell might also be able to generate negative refraction, as suggested in EM waves. ${ }^{7}$ Such a structure was originally suggested by Karathanos et $a l .{ }^{16}$ as an artificial helical material that can be used for rotating the plane of polarization of linearly polarized light. Here, we propose a chiral structure with two spheres per two-dimensional unit cell so that more flexibility is allowed to generate the proposed results in elastic waves. The paper is arranged as follows. In Sec. II, we will describe the structure of the chiral phononic crystal. In Sec. III, we will show the existence of polarization gaps in such structures. In Sec. IV, we will demonstrate that the negative group-velocity bands can be found in the chiral structure embedded with resonators. In Sec. V, we will give the conclusions.

\section{CHIRAL PHONONIC CRYSTAL}

The method of calculation is a layer multiple scattering theory (MST), which was formulated by Sainidou et al. ${ }^{17}$ and extended by Chen et al. ${ }^{18}$ The method was used to calculate transmission properties and band structures of phononic crystals made of layers that consist of two kinds of nonoverlapping spheres in the two-dimensional unit cell of each layer. ${ }^{18,19}$ Here we consider a phononic crystal generated by layers of spheres arranged in a hexagonal lattice [Fig. 1(a)] with a basis of two spheres. We start with the primitive vectors $\vec{a}_{1}=(1,0,0) a_{0}$ and $\vec{a}_{2}=(1 / 2, \sqrt{3} / 2,0) a_{0}$, where $a_{0}$ is the lattice constant of the structure. The two identical spheres of a basis are positioned as $(0,0,0)$ and $\left(0.5 a_{0}, 0,0\right)$ and this forms one layer. The successive layers are formed using the operation $\{R \mid T\}$, where the rotation $R$ is an anticlockwise $120^{\circ}$ rotation and $T=(0,0, d)$. The structure repeats itself after every three layers in the vertical direction, and the structure of the first, second, and third layers are depicted in Figs. $1(\mathrm{~b})-1(\mathrm{~d})$, respectively. The chiral phononic crystal is de- 


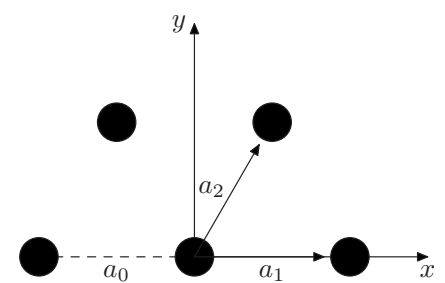

(a)
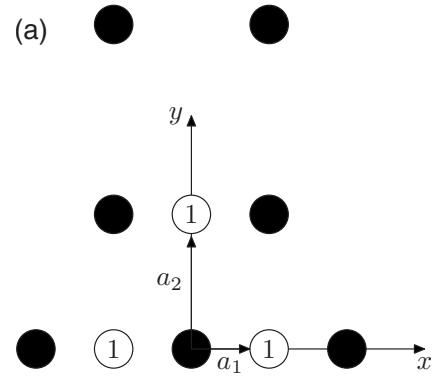

(b)
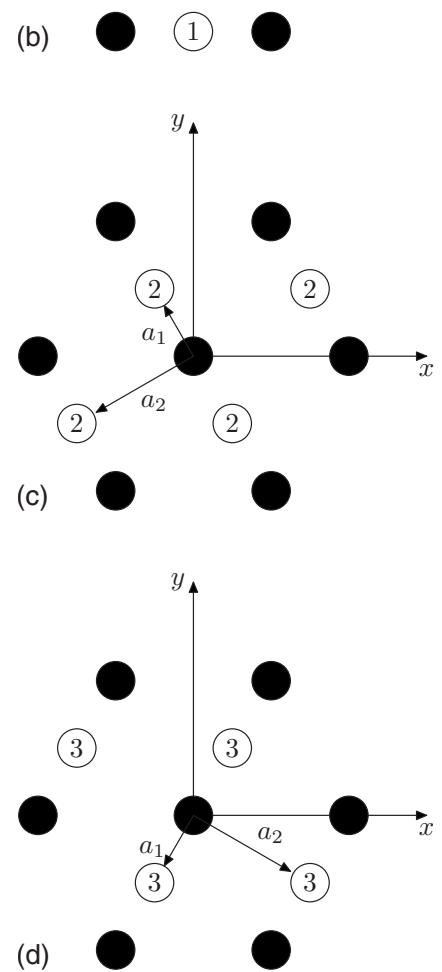

FIG. 1. Schematic structure of the repeating unit of the chiral phononic crystal. (a) The original hexagonal lattice. (b) The first layer in each repeating unit. (c) The second layer. (d) The third layer.

fined to be of the left-hand chirality. One can also generate the same structure of the right-hand chirality.

\section{POLARIZATION GAPS IN CHIRAL PHONONIC CRYSTAL}

In order to provide concrete numerical results to show the existence of polarization gaps, we consider mercury spheres ${ }^{20}$ of radius $S=0.2 a_{0}$ or $S=0.245 a_{0}$ embedded in an
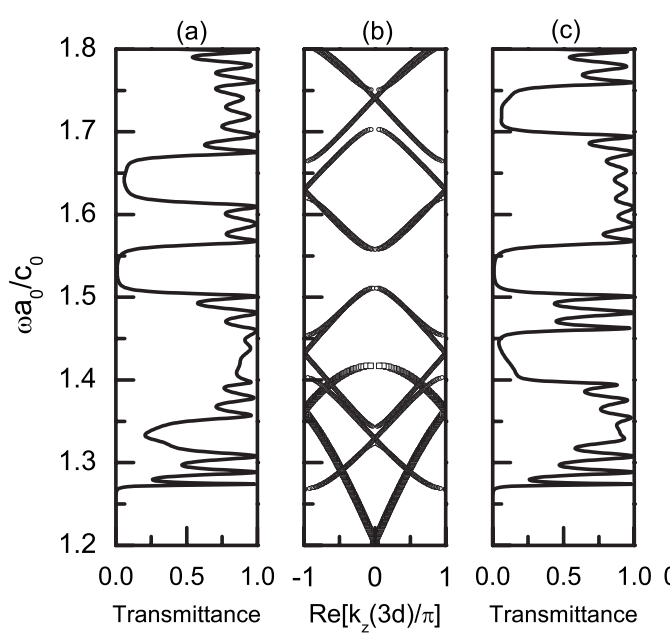

(d)

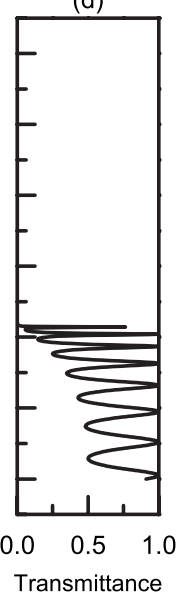

FIG. 2. Properties of the chiral phononic crystal with $d=3 a_{0}$ and $S=0.2 a_{0}$. (a) Transmission spectrum through four periods for lefthand CPT wave at normal incidence. (b) Band structure in the $z$ direction; the open square symbols correspond to the longitudinal mode, while the open circle symbols represent the transverse modes. (c) The same as in (a) but for right-hand CPT wave. (d) The same as in (a) but for longitudinal wave.

epoxy host matrix. For mercury, the relevant parameters are $\rho=13.5 \times 10^{3} \mathrm{~kg} / \mathrm{m}^{3}$ and $c_{l}=1450 \mathrm{~m} / \mathrm{s}$, and for epoxy, $\rho$ $=1.18 \times 10^{3} \mathrm{~kg} / \mathrm{m}^{3}, c_{l}=2540 \mathrm{~m} / \mathrm{s}$, and $c_{t}=1160 \mathrm{~m} / \mathrm{s}$. The results below are presented in normalized dimensionless units, ${ }^{17}$ where $c_{0}$ is set to $2540 \mathrm{~m} / \mathrm{s}$.

For the first example, the distance between each layer and radii of spheres is set at $d=3 a_{0}$ and $S=0.2 a_{0}$. The corresponding filling ratio is $2.6 \%$. Figures $2(\mathrm{a}), 2(\mathrm{c})$, and $2(\mathrm{~d})$ show, respectively, the transmittance curves of a slab with 12 layers in the $z$ direction at normal incidence for left-hand CPT elastic wave, right-hand CPT elastic wave, and longitudinal elastic wave. The corresponding band structure of the infinite chiral phononic crystal along the $z$ direction is shown in Fig. 2(b) for comparison. This example illustrates the properties of a low filling ratio limit of such systems.

From Fig. 2(d), we see that the longitudinal incident wave cannot propagate through the crystal at frequencies above $\omega a_{0} / c_{0} \sim 1.4$; i.e., longitudinal wave band gap exists in this region. After comparing both the transmission band region and the density of Fabry-Pérot peaks with the calculated band structure, the longitudinal mode [open square symbols in Fig. 2(b)] was identified. The eigenmodes of the chiral crystal are, in general, a hybrid between longitudinal and transverse modes. However, the normal incident waves (either longitudinal waves or transverse waves) will only excite modes of the same type in the interior of the crystal in the frequency range that $\omega a_{0} / c_{l}$ (epoxy) $<2 \pi$ (i.e., $\omega a_{0} / c_{0}<2 \pi$ ) for longitudinal waves [and $\omega a_{0} / c_{t}$ (epoxy) $<2 \pi$ (i.e., $\left.\omega a_{0} / c_{0}<2.87\right)$ for transverse waves]. ${ }^{21}$ So in the frequency range in Fig. 2, we observe no coupling between the longitudinal mode and the transverse $(T)$ modes. The chiral structure causes the $T$ modes to split into two branches. In general, each branch corresponds to one elliptically polarized mode, but it becomes circularly polarized near the band gaps. By comparing the band structure with the transmittance 


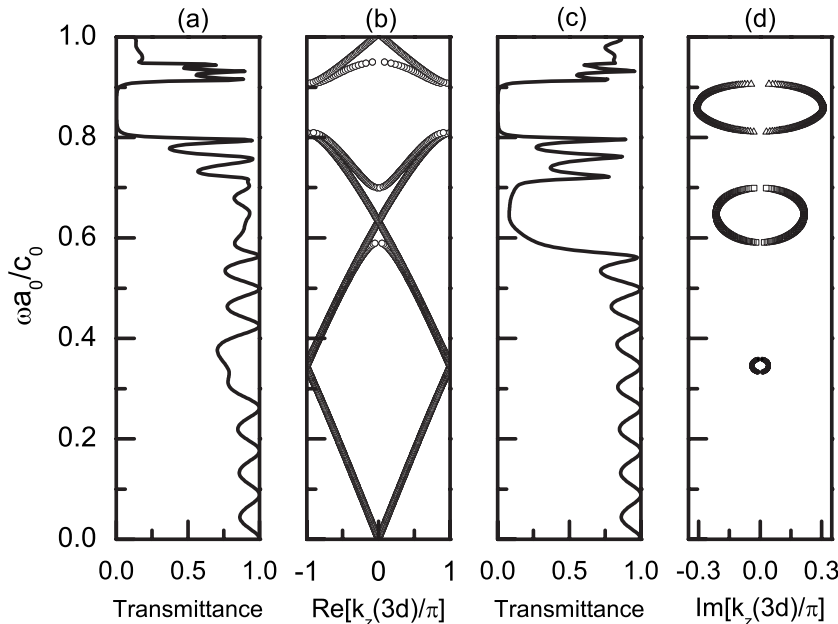

FIG. 3. Properties of the chiral phononic crystal with $d=0.5 a_{0}$ and $S=0.245 a_{0}$. (a) Transmission spectrum through four periods for left-hand CPT wave at normal incidence. (b) Band structure in the $z$ direction. (c) The same as in (a) but for right-hand CPT incident wave. (d) The imaginary part of the relevant complex band structure. The solid circle symbols and the open square symbols correspond to left-hand and right-hand polarization gaps respectively, while the open triangle symbols correspond to an absolute gap in which more polarizations are forbidden.

curves for CPT waves, a series of polarization gaps are identified for different polarizations. From Fig. 2(a), we find that the left-hand CPT wave is blocked near $\omega a_{0} / c_{0}=1.32$ $\sim 1.35, \omega a_{0} / c_{0}=1.5 \sim 1.55$, and $\omega a_{0} / c_{0}=1.62 \sim 1.67$. From Fig. 2(c), we find that the right-hand CPT wave is blocked near $\omega a_{0} / c_{0}=1.4 \sim 1.45, \omega a_{0} / c_{0}=1.5 \sim 1.55$, and $\omega a_{0} / c_{0}$ $=1.7 \sim 1.75$. The left-hand and right-hand CPT waves are both blocked near $\omega a_{0} / c_{0}=1.5 \sim 1.55$. We call the gaps associated only with the left-hand (right-hand) CPT waves the left-hand (right-hand) gaps. For gaps that block both CPT waves, they are absolute gaps. The sequence is left/right/ absolute, which is similar to the results of the EM waves. ${ }^{3,12}$ Similar results in the frequency range $\omega a_{0} / c_{0}<1.2$ (not shown) were also obtained. However, the effect due to chirality is very weak for this low-filling ratio structure in lower-frequency range. We will see in the following that the chiral effect is more apparent in the lower frequencies if we increase the filling ratio.

As a second example in Fig. 3, we consider a case in which the interlayer distances are smaller, and we also increase the sphere radii so that we have a higher filling ratio. When we set the distance between layers $d=0.5 a_{0}$, and the radius of the sphere $S=0.245 a_{0}$ (i.e., the filling ratio $=28.5 \%$ ), the polarization gaps are then enlarged. The longitudinal mode is not plotted here for clarity. From Fig. 3(a), we find that the left-hand CPT wave is slightly blocked near $\omega a_{0} / c_{0}=0.33$ and substantially blocked near $\omega a_{0} / c_{0}=0.8$ $\sim 0.9$. From Fig. 3(c), we find that the right-hand CPT wave is blocked near $\omega a_{0} / c_{0}=0.56 \sim 0.7$ and $\omega a_{0} / c_{0}=0.8 \sim 0.9$. The left- and right-hand CPT waves share the first absolute gap $\omega a_{0} / c_{0}=0.8 \sim 0.9$ in view of band structure in Fig. 3(b). The first left-hand gap occurs near $\omega a_{0} / c_{0}=0.33$, and the first right-hand gap occurs near $\omega a_{0} / c_{0}=0.56 \sim 0.7$. The band-gap sequence is also left/right/absolute. Another interesting property is that the center frequency of the first righthand gap is about twice of the center frequency of the first left-hand gap. It is due to the special discrete spiral symmetry of the chiral structure. At low frequency, the wavelengths of both left-hand and right-hand waves are much larger than the structural period. The bands are thus almost degenerate at low frequencies. As we gradually go from the low-frequency regime to a high-frequency regime, the wavelength of both the left-hand and right-hand waves decrease and the waves begin to "see" the chiral nature of the crystal. Let us first refer to Fig. 1(b), which shows the structure in one layer. We note that the packing density is higher along the $x$ direction and the packing density is lower along the $y$ direction. For the sake of simplicity, we will label the "density" direction as $\vec{a}_{1}$ and the "sparse" direction as $\vec{a}_{2}$. We see from Figs. $1(\mathrm{~b})-1(\mathrm{~d})$ that in the chiral structure, the vectors $\vec{a}_{1}$ and $\vec{a}_{2}$ are rotating as we go up the $z$ axis. At a frequency range that both waves having wavelength close to twice the structure's period (i.e., at the first zone boundary), the waves with different polarizations will be influenced by the materials differently. Let us compare the left-hand and right-hand waves at a fixed wavelength (in the crystal), which is equal to twice the structure's period [i.e., fixed $k(3 d)=\pi$ ]. At this $k$ point, the left-hand wave can have two solutions. The first left-hand wave can propagate in the crystal with transverse displacement field parallel to the $\vec{a}_{2}$ direction (sparse axis) of each layer. In that case, the wave will experience a lower effective refractive index. Alternatively, the second left-hand wave solution, which has its transverse displacement field parallel to the $\vec{a}_{1}$ "dense" axis of each layer, will experience a higher effective refractive index. Therefore, at a fixed wavelength inside the material, the first (second) left-hand wave has a higher (lower) frequency. We thus see two solutions for the left-hand wave at the same $k$ point. This explains why we have the first left-hand gap (first gap). In contrary, the righthand wave solution is intrinsically incommensurate with the left-hand structure and cannot have these two different solutions, so we do not have a right-hand gap here. When the wavelength is reduced to approximately a half of the waves near the first gap (i.e., almost at a double frequency), the situations for the two polarizations will be exchanged. In this case, we have a right-hand gap (second gap) but not a lefthand gap. When the wavelength is reduced to approximately a third of the waves near the first gap (i.e., almost at a triple frequency), both left-hand and right-hand gaps experience the crystal in the same way. Therefore, the existence of gap does not depend on polarization. This crystal has a repeating $\cdots A B C A B C \cdot \cdots$ structure. This nonhomogeneous periodicity gives the third band gap, which is independent of transverse polarizations.

The first left-hand gap is rather small, so the transmittance is not entirely suppressed in a 12-layer sample. To prove that the first left-hand gap does exist, we plot the imaginary part of the corresponding complex band structure of Fig. 3(b) in Fig. 3(d). The solid circle symbols show the nonzero $\operatorname{Im}\left(k_{z}\right)$ over the first left-hand-gap region, indicating the existence of the gap. The open square symbols [showing $\operatorname{Im}\left(k_{z}\right)$ of the first right-hand gap] confirm that the width of the gap is larger than the one of the left-hand gap. The open triangle 

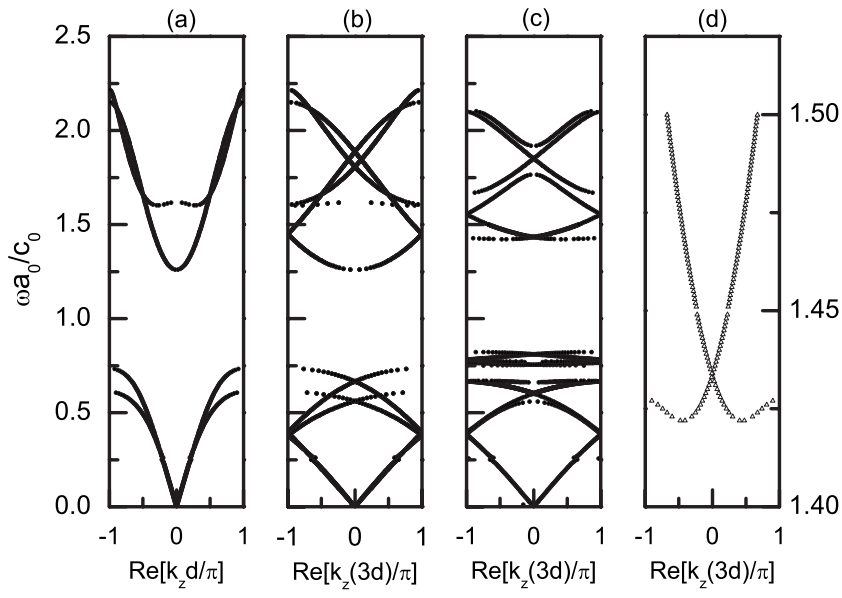

FIG. 4. Band structures with less resonance. (a) Nonchiral structure. (b) Same as (a) but using the reduced-zone scheme. (c) Chiral structure. (d) The first pass band above the resonance band gap in (c).

symbols also show the $\operatorname{Im}\left(k_{z}\right)$ of the first absolute gap. If we use 24 layers as a slab to scan the transmission spectrum with higher precision (results not shown), the transmittance within the first left-hand gap is then substantially suppressed. When the filling ratio is large enough, the chiral splitting of $T$-mode bands will be observable in the long-wavelength limit. One interesting feature we note in Fig. 2 and Fig. 3 is that two bands of the same symmetry can cross each other without opening a gap at the zone center or the zone boundary. This is a special feature for vector waves.

\section{NEGATIVE GROUP VELOCITY IN CHIRAL PHONONIC CRYSTAL}

To demonstrate the existence of band with negative group velocity, we then consider the following materials. The purpose is to create a structure in which the left and/or right propagating waves couple with a local resonance. The composite material has an epoxy host matrix embedding gold spheres coated with rubber. The coated spheres have radii $S=0.245 a_{0}$. Such hard-core and soft-coating configuration is designed to create local resonances. The relevant material parameters are $\rho=19.5 \times 10^{3} \mathrm{~kg} / \mathrm{m}^{3}, c_{l}=3360 \mathrm{~m} / \mathrm{s}$, and $c_{t}$ $=1240 \mathrm{~m} / \mathrm{s}$ for Au; $\rho=1.3 \times 10^{3} \mathrm{~kg} / \mathrm{m}^{3}, c_{l}=1300 \mathrm{~m} / \mathrm{s}$, and $c_{t}=87 \mathrm{~m} / \mathrm{s}$ for rubber; and $\rho=1.18 \times 10^{3} \mathrm{~kg} / \mathrm{m}^{3}, \quad c_{l}$ $=2540 \mathrm{~m} / \mathrm{s}$, and $c_{t}=1160 \mathrm{~m} / \mathrm{s}$ for epoxy. The distance between each layer is $d=0.5 a_{0}$. We will consider two specific radii of the hard core $\left(0.2042 a_{0}\right.$ and $\left.0.2242 a_{0}\right)$ in order to see the effect of different resonance strengths.

First, we focus on the case with a small core radius of $0.2042 a_{0}$. Figure 4 (a) shows the band structure of a nonchiral structure in which there is no rotation between each layer; e.g., layers depicted in Fig. 1(a) are stacked directly over one another. For the sake of clarity, the longitudinal mode is not plotted here because it does not couple with the transverse ones. We note that there is a resonance induced spectral gap, located in the frequency range $\omega a_{0} / c_{0}=0.75 \sim 1.25$. Figure 4(b) is the same as Fig. 4(a) except that the unit cell in the $z$
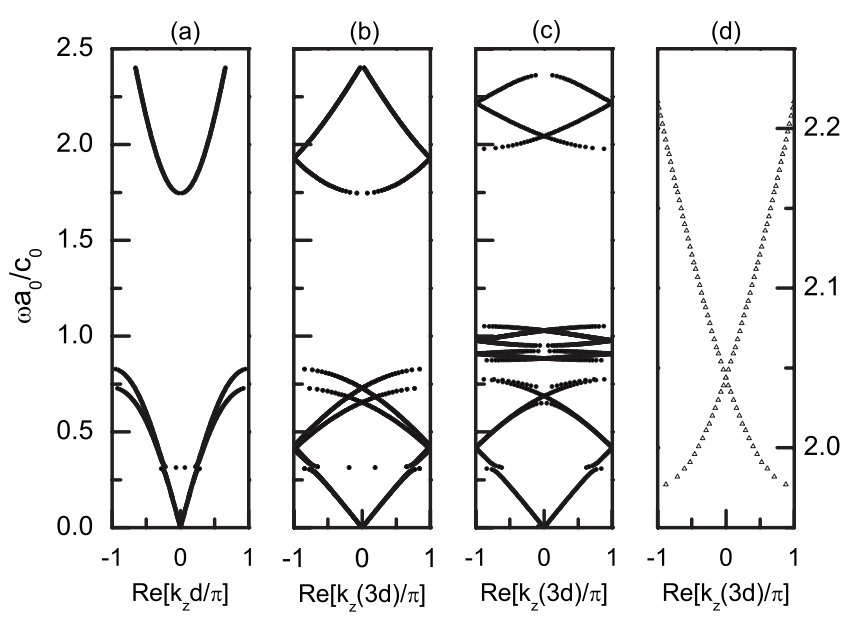

FIG. 5. Band structures with stronger resonance. (a) Nonchiral structure. (b) Same as (a) but using the reduced-zone scheme. (c) Chiral structure. (d) The first pass band above the resonance band gap in (c).

direction contains three layers, and we display the results using a reduced-zone scheme to see the band-folding effect so that we can make a direct comparison with the band structure of the chiral system shown in Fig. 4(c). For the chiral stacking, the band gap shifts slightly to $\omega a_{0} / c_{0}=0.8 \sim 1.4$. The most interesting effect is the emergence of a band of negative group velocity in the bottom of the first passband above the resonance band gap. The mechanism of such negative group velocity is in fact similar to that of the negative group velocity induced by chiral structure in EM waves, which has been discussed previously, ${ }^{1,2,7}$ and the result is a realization of such ideas in elastic waves. A magnified view of Fig. 4(c) is plotted in Fig. 4(d). The minimum of the first passband above the resonance band gap is neither at the zone center nor at the zone edge but at about $k_{z}(3 d) / \pi= \pm 0.5$. This gives a negative-group-velocity region $k_{z}(3 d) / \pi \in[$ $-0.5,0.5]$.

We then consider coated spheres with a bigger core radius of $0.2242 a_{0}$, which gives a stronger resonance, and the results are shown in Fig. 5. Figure 5(a) shows the band structure of the nonchiral structure, with the layers stacked directly over each other. The band gap is located in the range $\omega a_{0} / c_{0}=0.8 \sim 1.75$. It exhibits a stronger resonance with the width of the band gap significantly larger than that of the first case in Fig. 5(a). Figure 5(b) is the same as Fig. 5(a) but the unit cell contains three layers in the $z$ direction, and the band dispersion is displayed using a reduced-zone scheme. By using a bigger unit cell, we see the band-folding effect and this facilitates a direct comparison with Fig. 5(c), which shows the band structure of the chiral arrangement. In the chiral structure, the band gap shifts to $\omega a_{0} / c_{0}=1.1 \sim 1.9$, and a negative-group-velocity band appears in the bottom of the first passband above the resonance band gap. The difference induced by chirality can be seen when we compare Figs. 5(b) and 5(c). A magnified view of Fig. 5(c) is plotted in Fig. 5(d). When we compare Fig. 5(d) with Fig. 4(d), we see that a stronger resonance $[$ Fig. $5(\mathrm{c})]$ causes the minimum of the first passband above the resonance band gap shifts to the zone boundary so that the whole band exhibits negative 
group velocity. So, we see that the bandwidth of the negative-group-velocity band can be increased by increasing the strength of the resonant units.

\section{CONCLUSION}

In summary, we have investigated the properties of a chiral phononic crystal, which is constructed by rotating layers using the layer MST method. One basic property of such structure is the possibility of realizing various polarizations gaps in one single configuration. The polarization gaps are confirmed when comparing the transmittance curves and the corresponding band structure. The splitting of $T$ modes into left-hand mode and right-hand mode is observed and the effect can be enhanced in low-frequency range by increasing the filling ratio. It is found that the $L$ mode does not couple with the $T$ modes in low frequencies. We also demonstrated that when the chiral structure contains local resonators, the coupling of local resonance and CPT waves give rise to negative group velocity, with the bandwidth governed by the strength of the resonance, which is in turn governed by the size of the resonators. Our work can provide a more comprehensive comparison between elastic waves and EM waves, showing the linkage between structure and wave properties.

\section{ACKNOWLEDGMENTS}

This work was supported by Hong Kong RGC through Grant No. 600305. H.M. is supported by the National Natural Science Foundation of China under Grant No. 10334020 and the National Minister of Education Program for Changjiang Scholars and Innovative Research Team in University. Computation resources are supported by Shun Hing Education and Charity Fund.
${ }^{1}$ J. B. Pendry, Science 306, 1353 (2004).

${ }^{2}$ S. Tretyakov, Analytical Modeling in Applied Electromagnetics (Artech House, Norwood, 2003).

${ }^{3}$ J. C. W. Lee and C. T. Chan, Opt. Express 13, 8083 (2005).

${ }^{4}$ A. Chutinan and S. Noda, Phys. Rev. B 57, R2006 (1998).

${ }^{5}$ C. Oldano and S. Ponti, Phys. Rev. E 63, 011703 (2000).

${ }^{6}$ V. I. Kopp, V. M. Churikov, J. Singer, N. Chao, D. Neugroschl, and A. Z. Genack, Science 305, 74 (2004).

${ }^{7}$ V. Yannopapas, J. Phys.: Condens. Matter 18, 6883 (2006).

${ }^{8}$ J. C. W. Lee and C. T. Chan, Appl. Phys. Lett. 90, 051912 (2007).

${ }^{9}$ A. Lakhtakia, Sens. Actuators 80, 216 (2000).

${ }^{10}$ Jensen Li and C. T. Chan, Phys. Rev. E 70, 055602(R) (2004).

${ }^{11}$ V. C. Veselago, Sov. Phys. Usp. 10, 509 (1968).

${ }^{12}$ K. H. Fung, J. C. W. Lee, and C. T. Chan (unpublished).

${ }^{13}$ S. Yang, J. H. Page, Z. Y. Liu, M. L. Cowan, C. T. Chan, and P. Sheng, Phys. Rev. Lett. 93, 024301 (2004).

${ }^{14}$ N. G. Einspruch, J. Acoust. Soc. Am. 36, 971 (1964).

${ }^{15}$ Z. Y. Liu, X. X. Zhang, Y. Mao, Y. Y. Zhu, Z. Yang, C. T. Chan, and P. Sheng, Science 289, 1734 (2000).

${ }^{16}$ V. Karathanos, N. Stefanou, and A. Modinos, J. Mod. Opt. 42, 619 (1995).

${ }^{17}$ R. Sainidou, N. Stefanou, I. E. Psarobas, and A. Modinos, Comput. Phys. Commun. 166, 197 (2005).

${ }^{18}$ H. Y. Chen, X. D. Luo, and H. R. Ma, Phys. Rev. B 75, 024306 (2007).

${ }^{19}$ The program used here is a direct extension of the program package in Ref. 17, and the detailed modification is described in Ref. 18. All the structures here were calculated using the parameters $l=8$ and $19 \mathrm{~g}$ vectors (where $l$ is an angular-momentum cutoff and $g$ is the corresponding two-dimensional reciprocal lattice of the hexagonal lattice; for more details consult Refs. 17 and 18 to ensure the convergence).

${ }^{20}$ I. E. Psarobas and M. M. Sigalas, Phys. Rev. B 66, 052302 (2002).

${ }^{21}$ I. E. Psarobas, N. Stefanou, and A. Modinos, Phys. Rev. B 62, 278 (2000). 